\title{
Human Saliva Forms a Complex Film Structure on Alumina Surfaces
}

\author{
Marite Cárdenas, ${ }^{\dagger}$ Thomas Arnebrant, ${ }^{\dagger}$ Adrian Rennie, ${ }^{\ddagger}$ Giovanna Fragneto, ${ }^{\S}$ \\ Robert K. Thomas," and Liselott Lindh*,L \\ Surface Chemistry, Biomedical Laboratory Science, Faculty of Health and Society and Prosthetic Dentistry, \\ Faculty of Odontology, Malmö University, Malmö, Sweden, Material Physics Department, Uppsala \\ University, Uppsala, Sweden, Institut Laue-Langevin, Grenoble, France, Physical and Theoretical \\ Chemistry Laboratory, Oxford University, Oxford, United Kingdom
}

Received May 19, 2006; Revised Manuscript Received November 6, 2006

\begin{abstract}
Films formed from saliva on surfaces are important for the maintenance of oral health and integrity by protection against chemical and/or biological agents. The aim of the present study was to investigate adsorbed amounts, thickness, and structure of films formed from human whole saliva on alumina surfaces by means of in situ ellipsometry, neutron reflectivity, and atomic force microscopy. Alumina $\left(\mathrm{Al}_{2} \mathrm{O}_{3}\right.$, synthetic sapphire) is a relevant and interesting substrate for saliva adsorption studies as it has an isoelectric point close to that of tooth enamel. The results showed that saliva adsorbs rapidly on alumina. The film could be modeled in two layers: an inner and dense thin region that forms a uniform layer and an outer, more diffuse and thicker region that protrudes toward the bulk of the solution. The film morphology described a uniformly covering dense layer and a second outer layer containing polydisperse adsorbed macromolecules or aggregates.
\end{abstract}

\section{Introduction}

The oral cavity is an entrance for both beneficial and harmful substances into the human body. Human saliva is an essential body fluid, containing a range of protective components. Salivary films form readily on the surfaces present intra-orally. ${ }^{1}$ These films, denoted pellicles, protect oral surfaces from wear, dehydration, as well as demineralization. ${ }^{2}$ They are also known to influence bacterial binding. The protective functions are dependent on selective adsorption at natural or artificial surfaces as well as interactions taking place at these interfaces. The latter is dependent on the pellicle composition that affects the character of the biofilm, the so-called dental plaque. These processes influence the development of common oral diseases such as caries and periodontitis.

So far, many investigations dealing with adsorption from saliva and salivary proteins have been conducted on different types of model surfaces, mainly hydrophilic and hydrophobically modified silicon wafers with a surface that has been oxidized and an isoelectric point around $2 .^{3}$ Tooth enamel contains crystalline material (hydroxyapatite) present in prisms embedded in a protein matrix. The overall composition is hydroxyapatite $(95 \%)$, water $(4 \%)$, and enamel proteins $(1 \%) .{ }^{4}$ This type of surface is not available with the characteristics needed for most surface analysis techniques (flat, macroscopic surfaces with low roughness are required to follow adsorption phenomena with techniques such as neutron reflectivity, atomic force microscopy, and ellipsometry). Alumina $\left(\mathrm{Al}_{2} \mathrm{O}_{3}\right.$, synthetic sapphire, crystal plane 001 ) has an isoelectric point of $6.8,{ }^{5}$ which is close to

* Corresponding author. Tel.: +46 4066585 80. Fax: +46 4066585 03. E-mail: liselott.lindh@od.mah.se.

$\dagger$ Faculty of Health and Society, Malmö University.

$\doteqdot$ Uppsala University.

$\S$ Institut Laue-Langevin.

"Oxford University.

${ }^{\perp}$ Faculty of Odontology, Malmö University. what is expected for tooth enamel (approximately 7) and therefore is an interesting substrate for saliva adsorption studies as it may give a more clinically relevant picture of the saliva film structure. Studies of adsorption to this substrate, in combination with what it is known from experiments performed on other types of surfaces such as silica, will provide further insight into how surface properties influence the structure of the salivary film.

\section{Experimental Procedures}

Materials. Human whole saliva was collected from one healthy adult donor into a tube placed in a beaker filled with ice. The collection was done $2 \mathrm{~h}$ after breakfast on the day of the experiments as described by Dawes et al. ${ }^{6}$ The donor was considered to be in good oral health upon clinical examination. The sampling method used has recognized reproducible results ${ }^{7}$ as well as the fact that no statistically significant differences were found in adsorbed amounts of salivary components from a number of individual donors. ${ }^{8}$ The saliva was stored at $4{ }^{\circ} \mathrm{C}$ prior to usage and used without further treatment. The protein content of the saliva was determined to be $0.8 \mathrm{mg} \mathrm{mL}^{-1}$ using a BioRad Micro assay (Bio-Rad laboratories AB, Sundbyberg, Sweden), which is within the normal range. The committee on research ethics at Lund University has approved this study (LU 518-02). The buffer solution (denoted PBS) used was a $10 \mathrm{mM}$ phosphate buffer supplemented with $50 \mathrm{mM} \mathrm{NaCl}$ (pH 7.0). All water used was of ultrahigh quality (UHQ), processed in Elgastat UHQ II (Elga Ltd., High Wycombe, Bucks, England). Heavy water was provided from the reactor at ILL. Alumina surfaces (crystal plane 001) were obtained from MTI Corporation (Richmond, CA).

Methods. An automated Rudolf Research thin-film ellipsometer (type 43603-200E) was used as described in detail earlier ${ }^{9}$ to simultaneously measure the mean thickness and refractive index of the adsorbed layer in situ. The optical properties of the surface are characterized at the beginning of the adsorption experiment as described by Landgren and Jönsson. ${ }^{10}$ Then, the sample was injected by a 0.5 $\mathrm{mL}$ aliquot onto $4.5 \mathrm{~mL}$ of PBS buffer. The wavelength of the light used was $442.9 \mathrm{~nm}$ with an angle of incidence of $\sim 68^{\circ}$. The $5 \mathrm{~mL}$ 
optical glass cuvette (Hellma, Mülheim, Germany) was thermostated at $25.0 \pm 0.1{ }^{\circ} \mathrm{C}$, and the solution was agitated with a magnetic stirrer at $325 \mathrm{rpm}$. Rinsing of the cuvette was done at $15 \mathrm{~mL} / \mathrm{min}$. The recorded ellipsometric angles were evaluated using a three layer optical model, assuming isotropic media and planar interfaces. The mean refractive index, $n_{\mathrm{f}}$, and the ellipsometric thickness, $d_{\mathrm{f}}$, of the adsorbed layer were calculated numerically as described elsewhere. ${ }^{9,11}$ The adsorbed amount, $\Gamma$, was calculated from $n_{\mathrm{f}}$ and $d_{\mathrm{f}}$ using the formula

$$
\Gamma=\frac{\left(n_{\mathrm{f}}-n_{\mathrm{o}}\right) d_{\mathrm{f}}}{\mathrm{d} n / \mathrm{d} c}
$$

where $n_{\mathrm{o}}$ is the refractive index of the bulk solution and $\mathrm{d} n / \mathrm{d} c$ is the refractive index increment as a function of bulk concentration. For saliva, a $\mathrm{d} n / \mathrm{d} c=0.165 \mathrm{~g} / \mathrm{cm}^{3}$ was used since this value is representative for glycoproteins as the ones present in human saliva. ${ }^{12}$

Neutron reflection experiments were carried out on the D17 reflectometer at the Institut Laue-Langevin in Grenoble, France. ${ }^{13}$ All measurements were made in time-of-flight mode, using a wavelength range of 2.2-19 $\AA$. The sample solution was contained in a PTFE trough clamped against the alumina surface with hollow metal plates that allow temperature equilibration by a circulating water bath set at $25 \pm 1{ }^{\circ} \mathrm{C}$. The cell has an inlet and outlet, allowing the change of contents in situ without exposing the surface to air. Prior to the adsorption of saliva, the alumina surface was characterized in $\mathrm{D}_{2} \mathrm{O}$, $\mathrm{H}_{2} \mathrm{O}$, and a $90-10 \%(\mathrm{v} / \mathrm{v}) \mathrm{D}_{2} \mathrm{O}-\mathrm{H}_{2} \mathrm{O}$ mixture. The sensitivity of neutron reflectivity to the layer structure depends on its scattering contrast to the surrounding media. The neutron scattering length density (SLD) is the sum of the scattering lengths for each nucleus within a molecule or moiety divided by its volume. ${ }^{14,15}$ The SLD is sensitive to the isotopic composition of the molecules with large differences between, for example, ${ }^{1} \mathrm{H}$ and ${ }^{2} \mathrm{H}(n)$, and it is possible to selectively match molecules or surfaces by, for instance, appropriate mixing of light and heavy water. This is known as contrast variation or contrast matching (i.e., see Ottewill ${ }^{16}$ ). At $90-10 \%(\mathrm{v} / \mathrm{v}) \mathrm{D}_{2} \mathrm{O}-\mathrm{H}_{2} \mathrm{O}$, the scattering length density of the solvent matches that of the alumina substrate. This mixture of water matched to alumina is a very sensitive contrast to the adsorbed salivary film that is mainly composed of hydrogenated proteins with a lower scattering length density. The experiment was performed in several steps: (i) the clean alumina surface was characterized. (ii) 10 (v/v) \% HWS solution in PBS was added to the cell and left to adsorb. Data were acquired every $3 \mathrm{~h}$ until no significant changes in the reflectivity profile were observed. (iii) Extensive rinsing with PBS was performed, and finally desorption measurements were executed until no further change in the reflectivity profile was observed.

AFM imaging in a liquid cell was performed using a scanning probe microscope (Picoforce multimode SPM with a Nanoscope IV control unit, Veeco, Woodbury, NY). A silicon nitride tip (type DNP, Veeco) with cantilever constant of $0.06 \mathrm{~N} / \mathrm{m}$ was used. Imaging was performed in soft-contact mode by fixing the surface load to the electrostatic repulsive barrier. In this way, the probe is not in direct contact with the surface, and imaging of soft layers is possible without alterations caused during the probing. After $3 \mathrm{~h}$ of HWS adsorption on the alumina surface, extensive rinsing with PBS was executed through the liquid cell. Imaging was performed at room temperature at the solid/liquid interface. The height images were subjected to first-order flattening to remove the offset and tilt of each line using the software provided by Veeco. AFM imaging of HWS on alumina was performed twice on two different alumina slides.

\section{Results and Discussion}

Ellipsometric measurements performed neglecting a possible influence by birefringence ${ }^{9,10,17}$ on alumina surfaces indicate a rapid adsorption process from (10\%) human whole saliva (HWS) in $10 \mathrm{mM}$ phosphate buffer $\mathrm{pH} 7$ containing $50 \mathrm{mM} \mathrm{NaCl}$ at

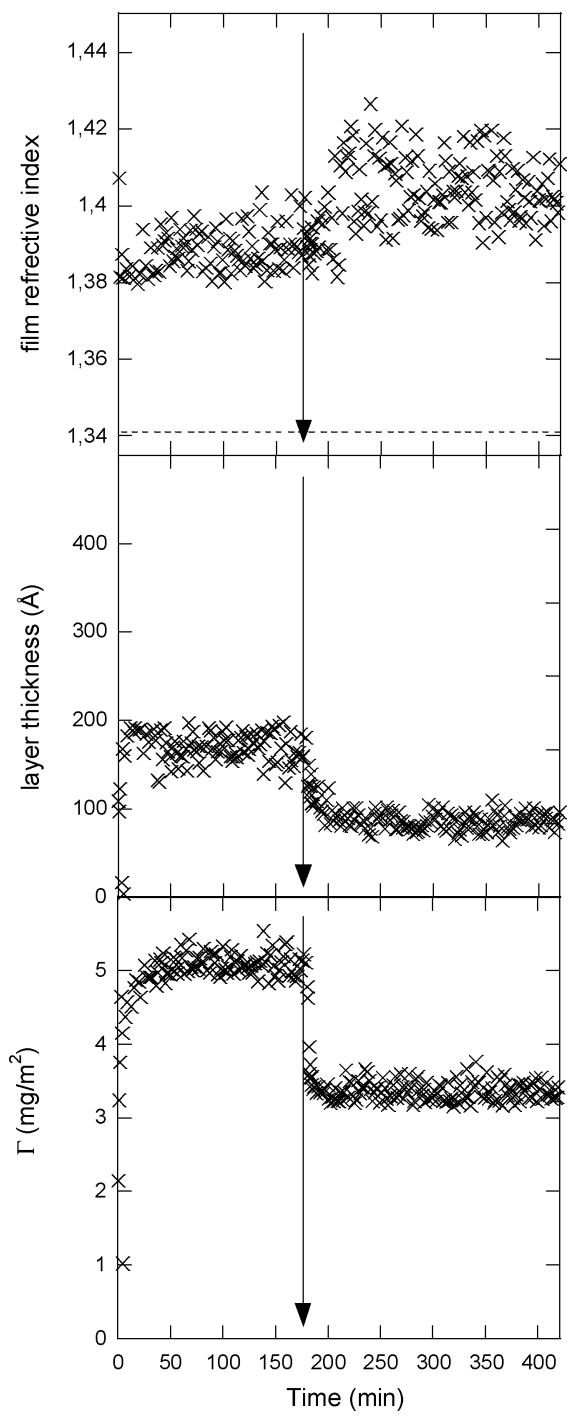

Figure 1. Film refractive index, film thickness $(\AA)$, and adsorbed amount $\left(\Gamma, \mathrm{mg} / \mathrm{m}^{2}\right)$ as a function of elapsed time for the adsorption from $10 \%(\mathrm{v} / \mathrm{v})$ human whole saliva to alumina. The arrow indicates the point at which rinsing with buffer solution was initiated. The broken line in the upper figure indicates the refractive index of the bulk solution.

$25{ }^{\circ} \mathrm{C}$ under continuous agitation (see Figure 1). Both the adsorbed amount $\left(5.4 \mathrm{mg} / \mathrm{m}^{2}\right)$ and the film thickness ( 200 $⿱$ A) reach steady-state conditions within $1 \mathrm{~h}$. After $3 \mathrm{~h}$ of adsorption time, extensive rinsing with buffer solution was performed that led to partial desorption of the adsorbed layer and a concomitant decrease in the layer thickness. The calculated film refractive index is also given in Figure 1 and shows changes in the film density upon rinsing with buffer. Four hours after rinsing, about $35 \%$ of the film in terms of surface excess had been removed from the surface. Interestingly, adsorption of HWS onto hydroxyapatite ${ }^{18}$ leads to a similar adsorption behavior in terms of initial kinetics and the size of the irreversibly bound portion of HWS (when subjected to extensive rinsing with buffer solution at conditions similar to those employed in the present work). The steady-state adsorbed amount after saliva addition (4 $\mathrm{mg} / \mathrm{m}^{2}$ ) was, however, slightly lower as compared to the adsorption on alumina. Similar results were obtained for adsorption of HWS on silica. ${ }^{7,18}$

Neutron reflectivity (NR) was used to analyze the structure of the salivary adsorbed layer on alumina surfaces. Figure 2 shows the neutron reflectivity profile for HWS films on alumina 


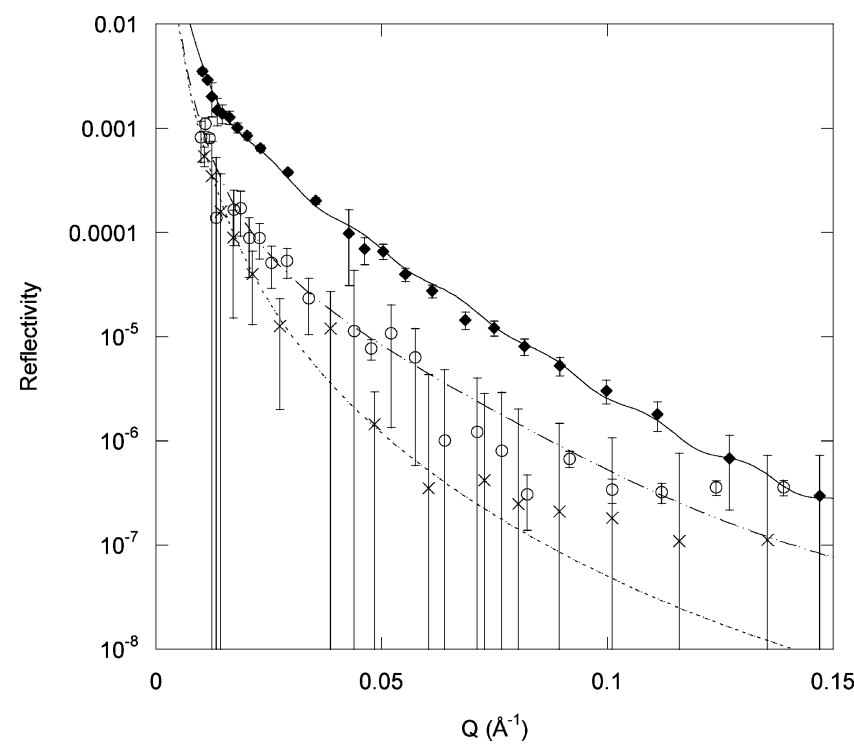

Figure 2. Neutron reflectivity curves (symbols) and fits (lines) to the data obtained for human whole saliva film onto an alumina surface after $12 \mathrm{~h}$ of equilibration (solid diamonds/solid line). The data for the bare block (crosses/dotted line) and for the remaining adsorbed layer after extensive rinsing (open circles/broken line) are included.

Table 1. Parameters Used for NR Fits Given in Figure $2^{a}$

volume

\begin{tabular}{llccc} 
sample & & thickness $(\AA)$ & SLD $_{\text {film }}\left(\AA^{-2}\right)$ & fraction $^{b}(\%)$ \\
\hline HWS & inner layer & 39 & $4.61 \times 10^{-6}$ & 38 \\
& outer layer & 285 & $5.61 \times 10^{-6}$ & 1.5 \\
rinsing & with buffer & 39 & $5.64 \times 10^{-6}$ & 11
\end{tabular}

a An inner roughness of $15 \AA$ for the bare alumina crystal was used in the fits (this value was obtained by fitting the NR data for three different contrasts of the bare surface). ${ }^{b}$ The volume fraction of the inner surface was calculated by assuming the SLD of the salivary material to be $2.9 \times$ $10^{-6} \AA^{-2}$. No roughness was added to the salivary adsorbed layers. Values are means and standard deviations of 10 different images and two separate experiments.

surfaces after $12 \mathrm{~h}$ of equilibration time. It also shows the data after the fluid cell was rinsed extensively with buffer solution and further equilibrated until no significant changes in the reflectivity profile were observed $(>12 \mathrm{~h})$. The specular reflectivity was calculated using an optical matrix model ${ }^{19}$ for a planar adsorbed film composed of homogeneous sublayers with a different scattering length density and thickness. The calculated reflectivity was then fitted to the experimental data using the computer program drydoc. ${ }^{20}$ Table 1 summarizes the parameters used in the fits shown in Figure 2. The scattering length density for the salivary film cannot be calculated precisely given that its molecular composition is quite complex. However, the salivary film is contrast matched when a $50-50 \%(\mathrm{v} / \mathrm{v})$ $\mathrm{H}_{2} \mathrm{O}-\mathrm{D}_{2} \mathrm{O}$ solvent mixture was used. Therefore, the corresponding SLD for the saliva should be close to $2.9 \times 10^{-6} \AA^{-2}$ even for solvents with a higher heavy water content (the $\mathrm{SLD}_{\text {saliva }}$ might be higher due to H/D exchange). This SLD value was then used to calculate the volume fraction of the salivary material within the adsorbed film. Although the obtained volume fractions are not strictly correct, they provide an idea of the proportions of the adsorbed material within the different sublayers.

On alumina, a slow adsorption process from HWS was observed using NR. After a few hours, a thin layer (40 ̊) covered the alumina surface with a volume fraction of $40 \mathrm{v} / \mathrm{v}$ $\%$ (data not shown). With time, a second layer developed that

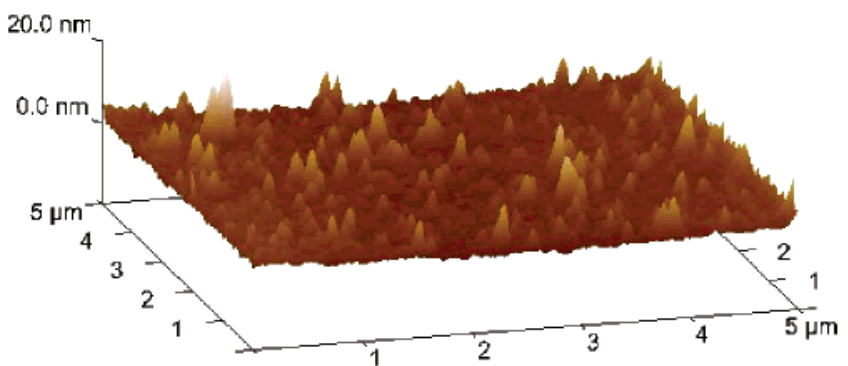

Figure 3. Surface plot obtained from the height image for a buffer eluted salivary film on alumina. The mean square surface roughness for the HWS film on alumina was $0.9 \pm 0.1 \mathrm{~nm}$ as compared to 0.21 $\pm 0.05 \mathrm{~nm}$ for the clean alumina surface (these values are averages of at least 10 different measurements).

was quite thick (300 $\AA$ ) and diffuse $(1.5 \mathrm{v} / \mathrm{v} \%)$. This layer stabilizes after approximately $12 \mathrm{~h}$ of equilibration time. The fact that a thin layer forms faster is in agreement with the general adsorption behavior of polydisperse polymers: the smaller, fast diffusing molecules adsorb initially to the surface. In a general case, these molecules are then replaced by large, slowly diffusing molecules with a higher surface affinity. ${ }^{21,22}$ Saliva consists of a mixture of proteins both globular and with flexible structures, among the latter statherins, histatins, and proline-rich proteins may be mentioned. Saliva also contains high molecular weight glycoproteins, mucins, which have a blocky structure with glycosylated and bare polypeptide segments. These large proteins are known to adsorb forming a very diffuse layer. ${ }^{23}$ For the salivary film, the volume fraction of the inner layer only slightly increases with time (data not shown), while the outer layer develops quite slowly. We interpret this as limited replacement of the smaller proteins in the inner layer possibly owing to the different natures of the proteins present in saliva.

Rinsing the salivary layer with buffer solution led to desorption, although it was larger for the NR experiments (Figure 2 and Table 1) as compared to ellipsometry (Figure 1). The NR fits indicate that the $\sim 300 \AA$ outer layer was removed completely upon rinsing with buffer while there was a significant decrease in the density of the inner layer. The fact that the steady-state values for the adsorbed amount in the neutron reflectivity experiments (compare Figure 1 and Table 1) are much lower than in the ellipsometric experiment could be due to various reasons. First, the neutron reflectivity profile does not depend only on the overall changes in the adsorbed amount and layer thickness (as ellipsometry does) but rather is sensitive to changes in the structure of the film. Indeed, the total volume fraction quickly stabilizes (a constant value is obtained after 3 $\mathrm{h}$ of equilibration time for the NR experiment). Second, the ellipsometric experiment was performed under continuous stirring, while the NR experiment lacked any agitation at all. This makes the mass transport and hydrodynamic conditions upon adsorption completely different for the ellipsometry experiments as compared to the NR one. Third, there is a significant difference between the substrate roughness for the NR and the substrate roughness for the ellipsometry/AFM experiments. The alumina blocks used for NR experiments presented a roughness as large as $15 \AA$ (Table 1), while the slides used for AFM and ellipsometry presented a roughness of $2 \AA$ (Figure 4). Finally, the surface area under study differs largely for the various techniques used: neutron reflectivity data were collected over a surface area of $\sim 10 \mathrm{~cm} \times 10 \mathrm{~cm}$, while the liquid cell used for AFM limits the surface area under study to $40 \mu \mathrm{m} \times 40 \mu \mathrm{m}$. A similar rationalization can be used to explain the difference in kinetics of adsorption/desorption in the NR experiment. 


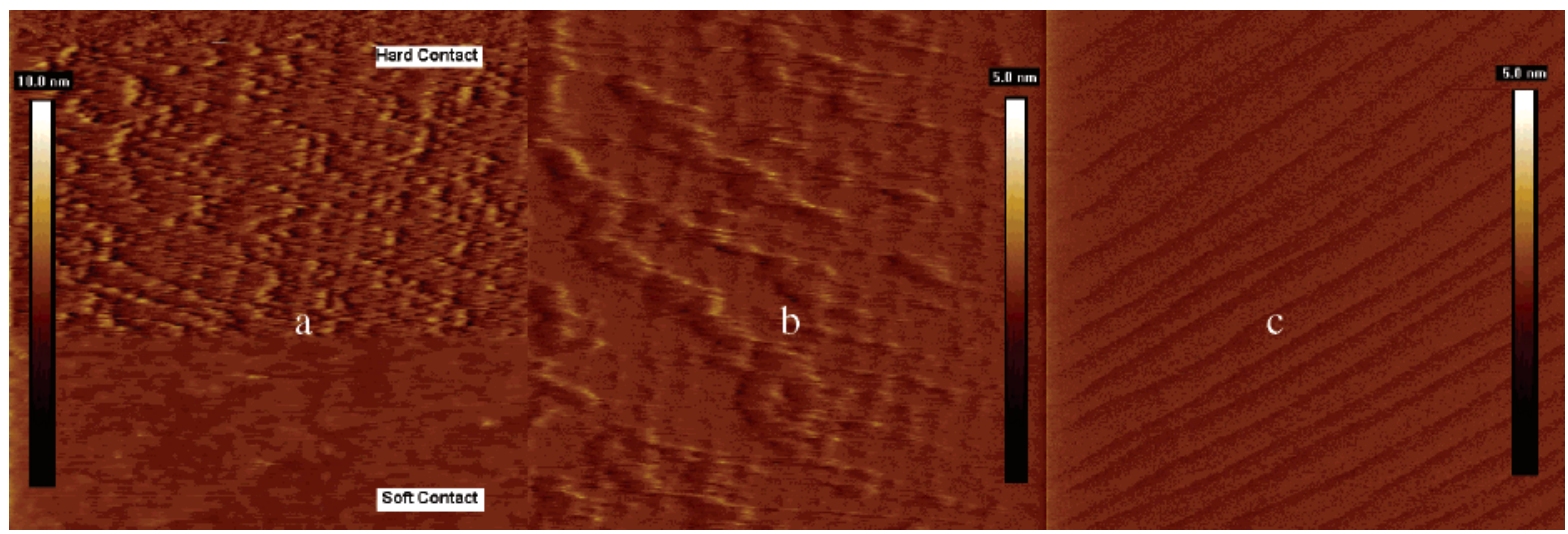

Figure 4. Deflection AFM images for a buffer eluted salivary film on alumina. The lower and upper parts of panel a are recorded using soft and hard contact mode imaging, respectively (the image represents an area of $2 \times 2 \mu \mathrm{m}^{2}$ ). Hard contact mode is used in panel b (HWS film) and panel c (clean surface prior to adsorption). The latter two images represent an area of $1 \times 1 \mu \mathrm{m}^{2}$.

Soft contact AFM imaging in liquids was performed on alumina surfaces in a liquid cell. This technique allows imaging of the proteinaceous adsorbed layer without major perturbations given that the interaction with the cantilever tip was set to the electrostatic repulsive regime. ${ }^{24,25}$ The salivary film could not be imaged prior to rinsing with buffer given the presence of large molecules/aggregates in the bulk and the high viscosity of the salivary solution. A typical surface plot (height image) obtained 30 min after rinsing with buffer is shown in Figure 3. No major changes in the morphology of the adsorbed layer could be detected with further elapsed time. Figure 3 clearly shows the complex morphology of the salivary film: there is a thin, denser layer that seems to uniformly cover the surface on top of which a few aggregates protrude toward the bulk. These adsorbed aggregates vary both in height (the maximum peak height is $110 \pm 36 \AA$ ) and width (the effect of the tip makes the interpretation of the width data complicated and therefore is not further discussed in this paper). Note from the image that the overall density of these aggregates is much lower as compared to that of the inner layer. Although the maximum height measured by AFM is lower than the outer diffuse layer found using NR, AFM corroborates the presence of two different types of sublayers: one denser close to the surface and another one diffuse protruding toward the bulk. In this regard, it must be mentioned that the $\sim 300 \AA$ outer layer determined by NR was not present in the film after extensive rinsing with buffer was performed, and therefore, it is not surprising that the maximum peak height measured by AFM is only $\sim 110 \AA$.

Interestingly, applying a hard contact force (within the compliance regime) cannot open a scratch in the film. This is shown in Figure 4a, which gives deflection images of the HWS film at two different imaging loads that correspond to soft and hard contacts. The hard contact reveals that the inner film has a network structure, in agreement with previous studies for bulk saliva. ${ }^{26}$ A detail of such network is given in Figure $4 \mathrm{~b}(1 \mu \mathrm{m}$ $\times 1 \mu \mathrm{m}^{2}$ ). For comparison, a deflection hard contact image of the clean alumina surface is given in Figure 4c. These results clearly indicate that the salivary layer is quite resistant toward mechanical stress.

In summary, saliva forms a complex pellicle on alumina surfaces composed of denser material near the surface and less dense near the solution. The total layer thickness of a salivary film formed on sapphire is found to be on the order of a few hundreds of Ångströms as measured by ellipsometry, NR, and AFM. These results are in accordance with findings in recently published studies of in vivo formed pellicles on enamel surfaces using transmission electron microscopy and $\mathrm{AFM},{ }^{27-29}$ where the authors also found that the in vivo salivary pellicle on enamel is composed of two types of layers: a basal layer on top of which larger proteins with a height of $300-400 \AA$ were adsorbed.

Acknowledgment. We are grateful to the Institut Laue Langevin, Grenoble for an allocation of neutron beam time. This study was supported by research grants from the Knowledge Foundation (KK-Stiftelsen, Biofilms, Research Centre for Biointerfaces), Malmö University, Stiftelsen Laryngfonden, The Swedish Dental Society, The Swedish Patent Revenue Fund for Research in Preventive Dentistry, The Swedish Research Council, and Uppsala University.

\section{References and Notes}

(1) Arnebrant, T. Protein adsorption in the oral environment. In Biopolymers at interfaces; Malmsten, M., Ed.; Marcel Dekker, Inc.: New York, 2003; pp 811-855.

(2) Lendenmann, U.; Grogan, J.; Oppenheim, F. G. Saliva and dental pellicle-a review. Adv. Dent. Res. 2000, 14, 22-28.

(3) Parks, G. A. The Isoelectric point of solid oxides, solid hydroxides, and aqueous hydroxo complex systems. Chem. Rev. 1965, 65, 177198.

(4) Jenkins, G. N. Saliva, chapter IX. In The physiology and biochemistry of the mouth; Blackwell Scientific Publishers: Oxford, 1978; pp 284359.

(5) Kershner, R. J.; Bullard, J. W.; Cima, M. J. Zeta potential orientation dependence of sapphire substrates. Langmuir 2004, 20, 4101-4108.

(6) Dawes, C.; Jenkins, G. N.; Tonge, C. H. The nomenclature of the integuments of the enamel surface of teeth. Br. Dent. J. 1963, 115, $65-68$.

(7) Vassilakos, N.; Arnebrant, T.; Glantz, P. O. Adsorption of whole saliva onto hydrophilic and hydrophobic solid surfaces. The influence of concentration, ionic strength, and pH. Scand. J. Dent. Res. 1992 , 100, 346-353.

(8) Lindh, L. et al. Concentration dependence of adsorption from human whole resting saliva at solid/liquid interfaces - an ellipsometric study. Biofouling 1999, 14 (3), 189-196.

(9) Azzam, R. M. A.; Bashara, N. M. Ellipsometry and polarized light; North-Holland: Amsterdam, 1977.

(10) Landgren, M.; Jönsson, B. Determination of the optical properties of $\mathrm{Si} / \mathrm{SiO}_{2}$ surfaces by means of ellipsometry, using different ambient media. J. Phys. Chem. 1993, 97, 1656-1660.

(11) Jenkins, T. E. Multiple angle-of-incidence ellipsometry. J. Phys D Appl. Phys. 1999, 32, 45-56.

(12) Jumel, K. et al. A polydisperse linear random coil model for the quaternary structure of pig colonic mucin. Eur. Biophys. J. 1997, $25,477-480$.

(13) Cubitt, R.; Fragneto, G. D17: the new reflectometer at the ILL. Appl. Phys. A 2002, 74, 329-331.

(14) Sears, V. F. Neutron Optics. In Neutron Optics; Oxford University Press: New York, 1989 
(15) Squires, G. L. Introduction to the theory of thermal neutron scattering; Dover Publications Inc.: Mineola, NY, 1978.

(16) Ottewill, R. H. Colloidal Dispersions, Special Publication 43; Royal Society of Chemistry: Cambridge, 1982.

(17) McCrackin, F. L. et al. Measurement of the thickness and refractive index of very thin films and the optical properties of surfaces by ellipsometry. J. Res. Natl. Bur. Stand. (U.S.) 1963, 67 (4), 363377

(18) Hahn Berg, I. C. et al. Salivary protein adsorption onto hydroxyapatite and SDS-mediated elution studied by in situ ellipsometry. Biofouling 2001, 17, 173-187.

(19) Born, M.; Wolf, E. Principles of optics. In Principles of optics. Pergamon: Elmsford, NY, 1970.

(20) Rennie, A. http://material.fysik.uu.se/Group_members/adrian/drydoc.htm.

(21) Fleer, G. J. et al. Polymer at interfaces, 1st ed.; Chapman and Hall: London, 1993.

(22) Vroman, L.; Adams, A. L. Adsorption of proteins out of plasma and solution in narrow spaces. J. Colloid Interface Sci. 1986, 111, 391402.
(23) Perez, E.; Proust, J. E. Forces between mica surfaces covered with adsorbed mucin across aqueous solution. J. Colloid Interface Sci. 1987, 118 (1), 182-191.

(24) Senden, T. J.; Drummond, C. J.; Kekicheff, P. Atomic force microscopy: imaging with electrical double layer interactions. Langmuir 1994, 10, 358-362.

(25) Manne, S. et al. AFM soft contact. Langmuir 1994, 10, 4409-4413.

(26) Wickstrom, C. et al. Macromolecular organization of saliva: identification of insoluble MUC5B assemblies and non-mucin proteins in the gel phase. Biochem. J. 2000, 351, 421-428.

(27) Hannig, M. et al. Atomic force microscopy study of salivary pellicles formed on enamel and glass in vivo. Colloid Polym. Sci. 2001, 279, 479-483.

(28) Hannig, M. et al. Transmission electron microscopy comparison of methods for collecting in situ formed enamel pellicle. Clin. Oral Invest. 2005, 9, 30-37.

(29) Schwender, N. et al. Initial bioadhesion on surfaces in the oral cavity investigated by scanning force microscopy. Appl. Surf. Sci. 2005, $252,117-122$

BM060492T 